\title{
The Self-Avoiding Walk: A Brief Survey*
}

\author{
Gordon Slade ${ }^{\dagger}$
}

\begin{abstract}
Simple random walk is well understood. However, if we condition a random walk not to intersect itself, so that it is a self-avoiding walk, then it is much more difficult to analyse and many of the important mathematical problems remain unsolved. This paper provides an overview of some of what is known about the critical behaviour of the self-avoiding walk, including some old and some more recent results, using methods that touch on combinatorics, probability, and statistical mechanics.
\end{abstract}

2010 Mathematics Subject Classification. Primary 60K35, 82B41.

Keywords. Self-avoiding walks, critical exponents, renormalisation group, lace expansion.

\section{Self-avoiding walks}

This article provides an overview of the critical behaviour of the self-avoiding walk model on $\mathbb{Z}^{d}$, and in particular discusses how this behaviour differs as the dimension $d$ is varied. The books $[29,40]$ are general references for the model. Our emphasis will be on dimensions $d=4$ and $d \geq 5$, where results have been obtained using the renormalisation group and the lace expansion, respectively.

An $n$-step self-avoiding walk from $x \in \mathbb{Z}^{d}$ to $y \in \mathbb{Z}^{d}$ is a map $\omega:\{0,1, \ldots, n\} \rightarrow$ $\mathbb{Z}^{d}$ with: $\omega(0)=x, \omega(n)=y,|\omega(i+1)-\omega(i)|=1$ (Euclidean norm), and $\omega(i) \neq \omega(j)$ for all $i \neq j$. The last of these conditions is what makes the walk self-avoiding, and the second last restricts our attention to walks taking nearestneighbour steps.

Let $d \geq 1$. Let $\mathcal{S}_{n}(x)$ be the set of $n$-step self-avoiding walks on $\mathbb{Z}^{d}$ from 0 to $x$. Let $\mathcal{S}_{n}=\cup_{x \in \mathbb{Z}^{d}} \mathcal{S}_{n}(x)$. Let $c_{n}(x)=\left|\mathcal{S}_{n}(x)\right|$, and let $c_{n}=\sum_{x \in \mathbb{Z}^{d}} c_{n}(x)=\left|\mathcal{S}_{n}\right|$. We declare all walks in $\mathcal{S}_{n}$ to be equally likely: each has probability $c_{n}^{-1}$. See Figure 1. We write $\mathbb{E}_{n}$ for expectation with respect to this uniform measure on $\mathcal{S}_{n}$.

What it is not:

- It is not the so-called "true" or "myopic" self-avoiding walk, i.e., the stochastic process which at each step looks at its neighbours and chooses uniformly from those visited least often in the past - the two models have different critical behaviour (see $[28,50]$ for recent progress on the "true" self-avoiding walk).

- It is by no means Markovian.

- It is not a stochastic process: the uniform measures on $\mathcal{S}_{n}$ do not form a consistent family.

\footnotetext{
*Revised May 28, 2010. To appear in Surveys in Stochastic Processes, Proceedings of the 33rd SPA Conference in Berlin, 2009, to be published in the EMS Series of Congress Reports, eds. J. Blath, P. Imkeller, S. Roelly.

${ }^{\dagger}$ Research supported in part by NSERC of Canada.
} 




Figure 1. A random self-avoiding walk on $\mathbb{Z}^{2}$ with $10^{6}$ steps. Illustration by T. Kennedy.

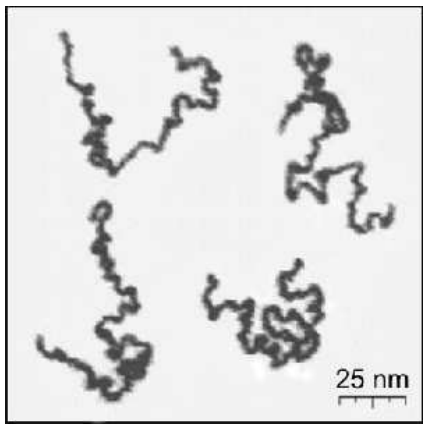

Figure 2. Appearance of real linear polymer chains as recorded using an atomic force microscope on surface under liquid medium. Chain contour length is $\approx 204 \mathrm{~nm}$; thickness is $\approx 0.4 \mathrm{~nm}$. [47]

\section{Motivations}

There are several motivations for studying the self-avoiding walk.

It provides an interesting and difficult problem in enumerative combinatorics: the determination of the probability of a walk in $\mathcal{S}_{n}$ requires the determination of $c_{n}$. It is also a challenging problem in probability, one that has proved resistant to the standard methods that have been successful for stochastic processes.

In addition, it is a fundamental example in the theory of critical phenomena in equilibrium statistical mechanics, and in particular is formally the $N \rightarrow 0$ limit of the $N$-vector model [15]. Finally, it is the standard model in polymer science of long chain polymers, with the self-avoidance condition modelling the excluded volume effect [14]. Figure 2 shows some 2-dimensional physical linear polymers, which may be compared with Figure 1. 


\section{Basic questions}

Three basic questions are to determine the behaviour of:

- $c_{n}=$ number of $n$-step self-avoiding walks,

- $\mathbb{E}_{n}|\omega(n)|^{2}=\frac{1}{c_{n}} \sum_{\omega \in \mathcal{S}_{n}}|\omega(n)|^{2}=$ mean-square displacement,

- the scaling limit, i.e., find $\nu$ and $X$ such that $n^{-\nu} \omega(\lfloor n t\rfloor) \Rightarrow X(t)$.

The inequality $c_{n+m} \leq c_{n} c_{m}$ follows from the fact that the right-hand side counts the number of ways that an $m$-step self-avoiding walk can be concatenated onto the end of an $n$-step self-avoiding walk, and such concatenations produce all $(n+m)$-step self-avoiding walks as well as contributions where the two pieces intersect each other. A consequence of this is that the connective constant $\mu=\lim _{n \rightarrow \infty} c_{n}^{1 / n}$ exists, with $c_{n} \geq \mu^{n}$ for all $n$ (see [40, Lemma 1.2.2] for the elementary proof). Since the $d^{n} n$-step walks that take steps only in the positive coordinate directions must be self-avoiding, we have $\mu \geq d$. And since the set of $n$-step walks without immediate reversals has cardinality $(2 d)(2 d-1)^{n-1}$ and contains all $n$-step self-avoiding walks, we have $\mu \leq 2 d-1$. Several authors have considered the problem of tightening these bounds. For example, for $d=2$ it is known that $\mu \in[2.625622,2.679193][33,46]$ and the non-rigorous estimate ${ }^{1}$ $\mu=2.63815853031(3)$ was obtained in [31].

Another basic question is to determine the behaviour of the two-point function $G_{z}(x)=\sum_{n=0}^{\infty} c_{n}(x) z^{n}$, when $z$ equals the radius of convergence $z_{c}=\mu^{-1}$ (see [40, Corollary 3.2.6] for a proof that $z_{c}=\mu^{-1}$ for all $x$ ). There is now a strong body of evidence in favour of the predicted asymptotic behaviours:

$$
c_{n} \sim A \mu^{n} n^{\gamma-1}, \quad \mathbb{E}_{n}|\omega(n)|^{2} \sim D n^{2 \nu}, \quad G_{z_{c}}(x) \sim c|x|^{-(d-2+\eta)},
$$

with universal critical exponents $\gamma, \nu, \eta$ obeying Fisher's relation $\gamma=(2-\eta) \nu$. The exponents are written as in (3.1) to conform with a larger narrative in the theory of critical phenomena. For $d=4$, logarithmic corrections are predicted: a factor $(\log n)^{1 / 4}$ should be inserted on the right-hand sides of the formulas for $c_{n}$ and $\mathbb{E}_{n}|\omega(n)|^{2}$ (but no logarithmic correction to the leading behaviour of $G_{z_{c}}(x)$ ). A prediction of universality is the statement that the critical exponents depend only on the dimension $d$ and not on fine details of how the model is defined. For example, the exponents are predicted to be the same for self-avoiding walks on the square, triangular and hexagonal lattices in two dimensions. This will not be the case for the connective constant or the amplitudes $A, D, c$, and for this reason the critical exponents have greater importance.

In the remainder of this paper, we discuss what has been proved concerning (3.1), dimension by dimension.

\footnotetext{
${ }^{1}$ The notation $\mu=2.63815853031(3)$ is an abbreviation, common in the literature, for $\mu=$ $2.63815853031 \pm 0.00000000003$.
} 


\section{Dimension $d=1$}

At first glance it appears that for $d=1$ the problem is trivial: $c_{n}=2$ and $|\omega(n)|=n$ for all $n$, so $\gamma=\nu=1$, and the walk moves ballistically left or right with speed 1.

However, the 1-dimensional problem is interesting for weakly self-avoiding walk. Let $g>0$, let $P_{n}$ be the uniform measure on all $n$-step nearest-neighbour walks $S=\left(S_{0}, S_{1}, \ldots, S_{n}\right)$ (with or without intersections), and let

$$
Q_{n}(S)=\frac{1}{Z_{n}} \exp \left[-g \sum_{i, j=0, i \neq j}^{n} \delta_{S_{i}, S_{j}}\right] P_{n}(S),
$$

where $Z_{n}$ is a normalisation constant.

Theorem 4.1. [17, 36] For every $g \in(0, \infty)$ there exists $\theta(g) \in(0,1)$ and $\sigma(g) \in$ $(0, \infty)$ such that

$$
\lim _{n \rightarrow \infty} Q_{n}\left(\frac{\left|S_{n}\right|-\theta n}{\sigma \sqrt{n}} \leq C\right)=\frac{1}{\sqrt{2 \pi}} \int_{-\infty}^{C} e^{-x^{2} / 2} d x .
$$

Note the ballistic behaviour for all $g>0$ : weakly self-avoiding walk is in the universality class of strictly self-avoiding walk. In particular, $\nu=1$, in contrast to $\nu=\frac{1}{2}$ for $g=0$. For any $g>0$, no matter how small, the 1-dimensional weakly self-avoiding walk behaves in the same manner as the strictly self-avoiding walk, which corresponds to $g=\infty$. This is predicted to be the case in all dimensions.

The proof of Theorem 4.1 is based on large deviation methods. For a different approach based on the lace expansion, see [25]. The natural conjecture that $g \mapsto$ $\theta(g)$ is (strictly) increasing remains unproved. For reviews of the case $d=1$, see $[26,27]$.

\section{Dimension $d=2$}

It was predicted by Nienhuis [44] that $\gamma=\frac{43}{32}$ and $\nu=\frac{3}{4}$ for $d=2$. According to Fisher's relation, this gives $\eta=\frac{5}{24}$. This prediction has been verified by extensive Monte Carlo experiments (see, e.g., [39]), and by exact enumeration plus series analysis. For the latter, $c_{n}$ is determined exactly for $n=1,2, \ldots, N$ and the partial sequence is analysed to determine its asymptotic behaviour. The finite lattice method is remarkable for $d=2$, where $c_{n}$ is known for all $n \leq 71$ [32]; in particular,

$$
c_{71}=4190893020903935054619120005916 \approx 4.2 \times 10^{30} .
$$

Concerning critical exponents and the scaling limit, a major breakthrough occurred in 2004 with the following result which connects self-avoiding walks and the Schramm-Loewner evolution (SLE). 
Theorem 5.1. [37] (loosely stated). If the scaling limit of the 2-dimensional selfavoiding walk exists and has a certain conformal invariance property, then the scaling limit must be $\mathrm{SLE}_{8 / 3}$.

Moreover, known properties of $\mathrm{SLE}_{8 / 3}$ lead to calculations that rederive the values $\gamma=\frac{43}{32}, \nu=\frac{3}{4}$, assuming that $\mathrm{SLE}_{8 / 3}$ is indeed the scaling limit [37]. The above theorem is a breakthrough because it identifies the stochastic process $\mathrm{SLE}_{8 / 3}$ as the candidate scaling limit. However, the theorem makes a conditional statement, and the existence of the scaling limit (and therefore also its conformal invariance) remains as a difficult open problem. Numerical verifications that $\mathrm{SLE}_{8 / 3}$ is the scaling limit were performed in [34].

Current results fall soberingly short of existence of the scaling limit and critical exponents for $d=2$. In fact, for $d=2,3,4$ the best rigorous bounds on $c_{n}$ are

$$
\mu^{n} \leq c_{n} \leq \begin{cases}\mu^{n} e^{C n^{1 / 2}} & (d=2) \\ \mu^{n} e^{C n^{2 /(d+2)} \log n} & (d=3,4)\end{cases}
$$

The lower bound comes for free from $c_{n+m} \leq c_{n} c_{m}$, and the upper bounds were proved in $[19,35]$. Worse, for $d=2,3,4$, neither of the inequalities $C^{-1} n \leq$ $\mathbb{E}_{n}|\omega(n)|^{2} \leq C n^{2-\epsilon}$ (for some $C, \epsilon>0$ ) has been proved. Thus, there is no proof that the self-avoiding walk moves away from its starting point at least as rapidly as simple random walk, nor sub-ballistically, even though it is preposterous that these bounds would not hold.

\section{Dimension $d=3$}

For $d=3$, there are no rigorous results for critical exponents. An early prediction for the values of $\nu$, referred to as the Flory values [14], was $\nu=\frac{3}{d+2}$ for $1 \leq d \leq 4$. This does give the correct answer for $d=1,2,4$, but it is not quite accurate for $d=3$. The Flory argument is very remote from a rigorous mathematical proof.

For $d=3$, there are three methods to compute the exponents. Field theory computations in theoretical physics [18] combine the $N \rightarrow 0$ limit for the $N$ vector model with an expansion in $\epsilon=4-d$ about dimension $d=4$, with $\epsilon=1$. Monte Carlo studies now work with walks of length 33,000,000 [12], using the pivot algorithm [41, 30]. Finally, exact enumeration plus series analysis has been used; currently the most extensive enumerations in dimensions $d \geq 3$ use the lace expansion [13], and for $d=3$ walks have been enumerated to length $n=30$, with the result $c_{30}=270569905525454674614$. The exact enumeration estimates for $d=3$ are $\mu=4.684043(12), \gamma=1.1568(8), \nu=0.5876(5)$ [13]. Monte Carlo estimates are consistent with these values: $\gamma=1.1575(6)[11]$ and $\nu=0.587597(7)$ $[12]$. 


\section{Dimension $d=4$}

7.1. The upper critical dimension. A prediction going back to [1] is that for $d=4$,

$$
c_{n} \sim A \mu^{n}(\log n)^{1 / 4}, \quad \mathbb{E}|\omega(n)|^{2} \sim D n(\log n)^{1 / 4} .
$$

Correspondingly, when the 4-dimensional self-avoiding walk is rescaled by the factor $(D n)^{-1 / 2}(\log n)^{-1 / 8}$, the scaling limit is predicted to be Brownian motion. The logarithmic corrections in (7.1) are typical of behaviour at the upper critical dimension, which is $d=4$ for the self-avoiding walk. As discussed in Section 8 below, self-avoiding walks behave like simple random walks in dimensions greater than 4.

A quick way to guess that 4 is the upper critical dimension is to recall that the ranges of two independent Brownian motions do not intersect each other if and only if $d \geq 4$, a fact intimately related to the 2-dimensional nature of Brownian paths. Consequently, one might guess that conditioning a simple random walk not to intersect itself might have no noticeable effect on the scaling limit when $d \geq 4$.

7.2. Continuous-time weakly self-avoiding walk. Let $X$ be the continuoustime simple random walk on $\mathbb{Z}^{d}$ with $\operatorname{Exp}(1)$ holding times and right-continuous sample paths. In other words, the walk takes its nearest neighbour steps at the events of a rate- 1 Poisson process. Let $\mathbb{E}_{x}$ denote expectation for this process started at $X(0)=x$. The local time at $x$ up to time $T$ is given by

$$
t_{x, T}=\int_{0}^{T} \mathbb{1}_{X(s)=x} d s
$$

and the amount of self-intersection experienced by $X$ up to time $T$ is measured by

$$
\int_{0}^{T} d s_{1} \int_{0}^{T} d s_{2} \mathbb{1}_{X\left(s_{1}\right)=X\left(s_{2}\right)}=\sum_{x \in \mathbb{Z}^{d}} t_{x, T}^{2}
$$

Let $g>0$ and $x \in \mathbb{Z}^{d}$. The continuous-time weakly self-avoiding walk two-point function is defined by

$$
G_{g, \lambda}(x)=\int_{0}^{\infty} \mathbb{E}_{0}\left(e^{-g \sum_{z \in \mathbb{Z}^{d}} t_{z, T}^{2}} \mathbb{1}_{X(T)=x}\right) e^{-\lambda T} d T,
$$

where $\lambda$ is a parameter (possibly negative) which is chosen in such a way that the integral converges. A subadditivity argument shows that there exists a critical value $\lambda_{c}=\lambda_{c}(g)$ such that $\sum_{x \in \mathbb{Z}^{d}} G_{g, \lambda}(x)<\infty$ if and only if $\lambda>\lambda_{c}$. The following theorem shows that the asymptotic behaviour of the critical two-point function has the same $|x|^{2-d}$ decay as simple random walk, i.e., $\eta=0$, in all dimensions greater than or equal to 4 , when $g$ is small. In particular, there is no logarithmic correction at leading order when $d=4$. 
Theorem 7.1. [8, 9] Let $d \geq 4$. There exists $\bar{g}>0$ such that for each $g \in(0, \bar{g})$ there exists $c_{g}>0$ such that as $|x| \rightarrow \infty$,

$$
G_{g, \lambda_{c}(g)}(x)=\frac{c_{g}}{|x|^{d-2}}(1+o(1)) \text {. }
$$

The proof of Theorem 7.1 is based on a rigorous renormalisation method $[8,9]$ (see also [2]), discussed further below.

7.3. Hierarchical lattice and walk. Theorem 7.1 has precursors for the weakly self-avoiding walk on a 4-dimensional hierarchical lattice. The hierarchical lattice is a replacement of $\mathbb{Z}^{d}$ by a recursive structure which is well-suited to the renormalisation group, and which has a long tradition of use for development of renormalisation group methodology.

The hierarchical lattice $\mathbb{H}_{d, L}$ ia a countable group which depends on two integer parameters $L \geq 2$ and $d \geq 1$. It is defined to be the direct sum of infinitely many copies of the additive group $\mathbb{Z}_{n}=\{0,1, \ldots, n-1\}$ with $n=L^{d}$. A vertex in the hierarchical lattice has the form $x=\left(\ldots, x_{3}, x_{2}, x_{1}\right)$ with each $x_{i} \in \mathbb{Z}_{n}$ and with all but finitely many entries equal to 0 . For $x \in \mathbb{H}_{d, L}$, let

$$
|x|= \begin{cases}0 & \text { if all entries of } x \text { are } 0 \\ L^{N} & \text { if } x_{N} \neq 0 \text { and } x_{i}=0 \text { for all } i>N\end{cases}
$$

A metric (in fact an ultra-metric) on $\mathbb{H}_{d, L}$ is then defined by

$$
\rho(x, y)=|x-y| \text {. }
$$

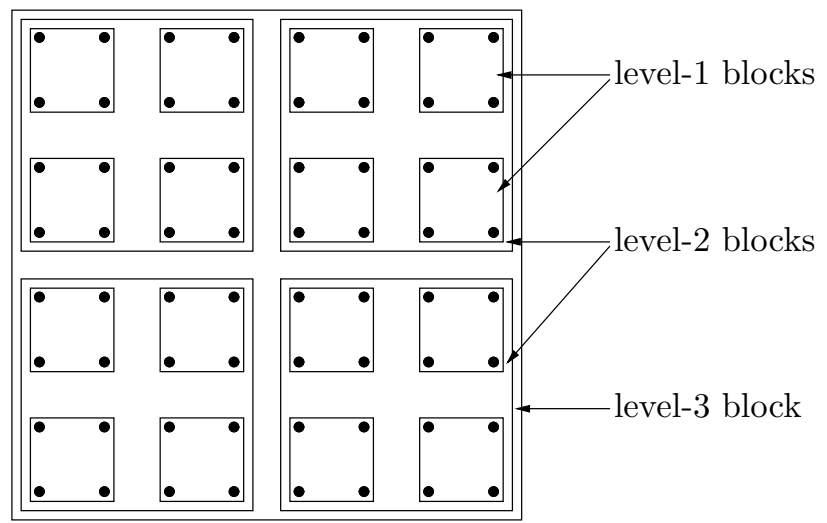

Figure 3. Vertices of the hierarchical lattice with $L=2, d=2$.

To visualise the hierarchical lattice, an example of the vertices of a finite piece of the $d$-dimensional hierarchical lattice with parameters $L=2$ and $d=2$ is depicted in Figure 3. Vertices are arranged in nested blocks of cardinality $L^{d j}$ where $j$ is 
the block level. Every pair of vertices is joined by a bond, with the bonds labelled according to their level as depicted in Figure 4. The level $\ell(x, y)$ of a bond $\{x, y\}$ is defined to be the level of the smallest block that contains both $x$ and $y$. The metric $\rho$ on the hierarchical lattice is then given in terms of the level by

$$
\rho(x, y)=L^{\ell(x, y)} .
$$

There is more structure present in Figure 3 than actually exists within the hierarchical lattice. In particular, all vertices within a single level-1 block are distance $L$ from each other, and their arrangement in a square in the figure has no relevance for the metric. With this in mind, the arrangement of the vertices as in Figure 3 serves to emphasise the difference between the hierarchical lattice and the Euclidean lattice $\mathbb{Z}^{d}$.

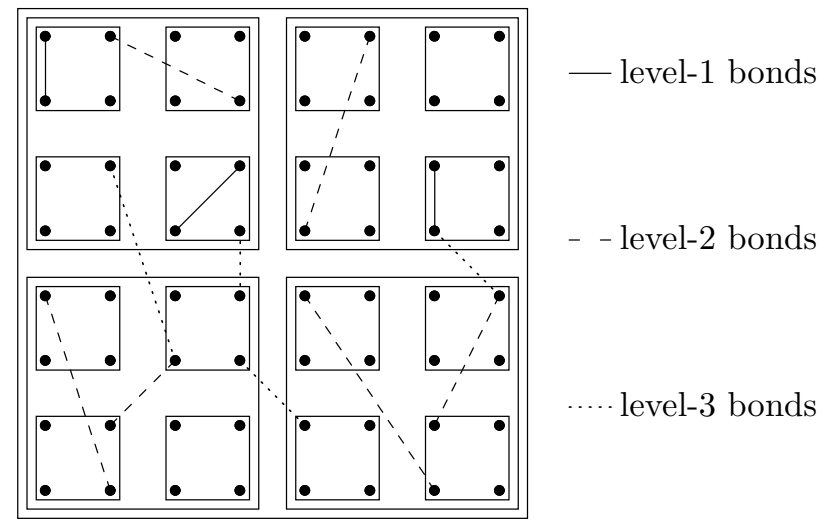

Figure 4. Bonds of the hierarchical lattice with $L=2, d=2$.

We now define a random walk on $\mathbb{H}_{d, L}$, in which the probability $P(x, y)$ of a jump from $x$ to $y$ in a single step is given by

$$
P(x, y)=\operatorname{const} \rho(x, y)^{-(d+2)} .
$$

We consider both the discrete-time random walk, in which steps are taken at times $1,2,3, \ldots$, and also the continuous-time random walk in which steps are taken according to a rate- 1 Poisson process. For the continuous-time process, the random walk Green function is defined to be

$$
G(x, y)=\int_{0}^{\infty} d T \mathbb{E}_{x}\left(\mathbb{1}_{X(T)=y}\right)
$$

where $\mathbb{E}_{x}$ denotes expectation for the process $X$ started at $x$. It is shown in [3] that for $d>2$

$$
G(x, y)=\operatorname{const} \frac{1}{\rho(x, y)^{d-2}} \quad \text { if } x \neq y,
$$


and in this sense the random walk on the hierarchical lattice behaves like a $d$ dimensional random walk.

The continuous-time weakly self-avoiding walk is defined as in Section 7.2, namely we modify the probability of a continuous-time random walk $X$ on $\mathbb{H}_{d, L}$ by a factor $\exp \left[-g \sum_{x} t_{x, T}(X)^{2}\right]$. The prediction is that for all $g>0$ and all $L \geq 2$ the weakly self-avoiding walk (with continuous or discrete time) on $\mathbb{H}_{d, L}$ has the same critical behaviour as the strictly self-avoiding on $\mathbb{Z}^{d}$ (at least for $d>2$ where (7.11) holds). This has been exemplified for $d=4$ in the series of papers $[3,5,6]$, where, in particular, the following theorem is obtained. There are some details omitted here that are required for a precise statement, and we content ourselves with a loose statement that captures the main message from [5].

Theorem 7.2. [5] (loosely stated). Fix $L \geq 2$. For the continuous-time weakly self-avoiding walk on the 4-dimensional hierarchical lattice $\mathbb{H}_{4, L}$, if $g \in\left(0, g_{0}\right)$ with $g_{0}$ sufficiently small, then there is a constant $c=c(g, L)$ such that

$$
\mathbb{E}_{0, g}^{T}|\omega(T)| \approx c T^{1 / 2}\left(\log _{L} T\right)^{1 / 8}\left[1+\frac{\log _{L} \log _{L} T}{32 \log _{L} T}+O\left(\frac{1}{\log _{L} T}\right)\right],
$$

where the expectation on the left-hand side is that of weakly self-avoiding walk started at 0 and up to time $T$, and where the symbol $\approx$ requires an appropriate interpretation; see [5, p. 525] for the details of this interpretation.

It is also shown in [3] that $\eta=0$ in the setting of Theorem 7.2. Very recently, related results for the critical two-point function and the susceptibility have been obtained in [21] for the discrete-time weakly self-avoiding walk on $\mathbb{H}_{4, L}$ with $g$ sufficiently small and $L$ sufficiently large. These results produce the predicted logarithmic correction for the susceptibility, closely related to (7.1).

The proofs of all these results for the 4-dimensional hierarchical lattice are based on renormalisation group methods, but very different approaches are used in $[3,5,6]$ and in $[21]$. The approach of $[21]$ is based on a direct analysis of the self-avoiding paths themselves. In contrast, the approach of $[3,5,6]$, as well as the proof of Theorem 7.1, are based on a functional integral representation for the two-point function with no direct path analysis.

7.4. Functional integral representation. The point of departure of the proofs of Theorems 7.1-7.2, and more generally of the analysis in [3, 5, 6, 8, 9, 16, 43], is a functional integral representation for self-avoiding walks. Such representations have their roots in $[45,42,38,3]$ and recently have been summarised and extended in [7]. We now describe the representation for the continuous-time weakly selfavoiding walk on $\mathbb{Z}^{4}$.

In fact, the representation is valid for weakly self-avoiding walk on any finite set $\Lambda$, and an extension to $\mathbb{Z}^{4}$ requires a finite volume approximation followed by an infinite volume limit; the latter is not discussed here. For the present discussion, let $\Lambda$ be an finite box in $\mathbb{Z}^{d}$, of cardinality $M$, and with periodic boundary conditions. Let $\Delta$ denote the lattice Laplacian on $\Lambda$. Let $X$ be the continuous-time Markov process on $\Lambda$ with generator $\Delta$, and let $\mathbb{E}_{x}$ denote the expectation for this process 
started from $x \in \Lambda$. We define the weakly self-avoiding walk two-point function on $\Lambda$ by

$$
G_{x, y}^{\mathrm{wsaw}, \Lambda}=\int_{0}^{\infty} \mathbb{E}_{x}\left(e^{-g \sum_{z \in \Lambda} t_{z, T}^{2}} \mathbb{1}_{X(T)=y}\right) e^{-\lambda T} d T,
$$

where $g>0$ and where $\lambda \in \mathbb{R}$ is chosen so that the integral converges.

Given $\varphi: \Lambda \rightarrow \mathbb{C}$, we write $\psi_{x}=\frac{1}{\sqrt{2 \pi i}} d \varphi_{x}$, where $d \varphi_{x}$ denotes the differential and we fix any particular choice of the square root. For $x \in \Lambda$, we define

$$
\tau_{x}=\varphi_{x} \bar{\varphi}_{x}+\psi_{x} \wedge \bar{\psi}_{x}
$$

where the wedge product is the usual anti-commuting product of differential forms, $\bar{\varphi}_{x}$ denotes the complex conjugate of $\varphi_{x}$, and $\bar{\psi}_{x}=\frac{1}{\sqrt{2 \pi i}} d \bar{\varphi}_{x}$. Forms are always multiplied using the wedge product, and we drop the wedge from the notation in what follows. We also define

$$
S=\sum_{x, y \in \Lambda}\left(-\Delta_{x, y}\right) \varphi_{x} \bar{\varphi}_{y}+\sum_{x, y \in \Lambda}\left(-\Delta_{x, y}\right) \psi_{x} \bar{\psi}_{y} .
$$

The integral representation for $G_{x, y}^{\mathrm{wsaw}, \Lambda}$ is

$$
G_{x, y}^{\mathrm{wsaw}, \Lambda}=\int e^{-S} e^{-\sum_{x \in \Lambda}\left(g \tau_{x}^{2}+\lambda \tau_{x}\right)} \bar{\varphi}_{x} \varphi_{y}
$$

where the integral is defined by the following procedure.

First, the integrand, which involves functions of differential forms, is defined by its formal power series about its degree-zero part. For example, with the abbreviated notation $S=-\varphi \Delta \bar{\varphi}-\psi \Delta \bar{\psi}$, the expansion of $e^{-S}$ is

$$
e^{-S}=e^{\varphi \Delta \bar{\varphi}+\psi \Delta \bar{\psi}}=e^{\varphi \Delta \bar{\varphi}} \sum_{N=1}^{|\Lambda|} \frac{1}{N !}(\psi \Delta \bar{\psi})^{N},
$$

where the sum is a finite sum due to the anti-commutativity of the wedge product. Second, in the expansion of the integrand, we keep only terms with one factor $d \varphi_{x}$ and one $d \bar{\varphi}_{x}$ for each $x \in \Lambda$, and discard the rest. Then we write $\varphi_{x}=u_{x}+i v_{x}$, $\bar{\varphi}_{x}=u_{x}-i v_{x}$ and similarly for the differentials, use the anti-commutativity of the wedge product to rearrange the differentials to $\prod_{x \in \Lambda} d u_{x} d v_{x}$, and finally perform the resulting Lebesgue integral over $\mathbb{R}^{2|\Lambda|}$. For further discussion and a proof of (7.16), see [7].

The approach of $[3,5,6,8,9]$ to the weakly self-avoiding walk is to study the integral on the right-hand side of (7.16), and simply to forget about the walks themselves. The differential form $e^{-S} e^{-V(\Lambda)}$, where $V(\Lambda)=\sum_{x \in \Lambda}\left(g \tau_{x}^{2}+\lambda \tau_{x}\right)$, has a property called supersymmetry (see [7] for a discussion of this in our context). In physics, roughly speaking, this corresponds to symmetry under an interchange of bosons and fermions. Supersymmetry has interesting consequences. For example, a general theorem (see $[6,7]$ ) implies that

$$
\int e^{-S} e^{-V(\Lambda)}=1
$$


We redefine $S$ as $S=\varphi(\epsilon I-\Delta) \bar{\varphi}-\psi(\epsilon I-\Delta) \bar{\psi}$ for some (small) choice of $\epsilon>0$, where $I$ denotes the $|\Lambda| \times|\Lambda|$ identity matrix. This can be regarded an adjustment of the parameter $\lambda$. Then, given a form $F$, we write

$$
\mathbb{E}_{C} F=\int e^{-S} F
$$

where $C=(\epsilon I-\Delta)^{-1}$. By (7.18), $\mathbb{E}_{C} 1=1$. We regard $\mathbb{E}_{C}$ as a mixed bosonicfermionic Gaussian expectation, with covariance $C$. The operation $\mathbb{E}_{C}$ has much in common with standard Gaussian integration, and for this reason we write $\mathbb{E}$ for expectation, but this is not ordinary probability theory and the expectations are actually Grassmannian integrals.

7.5. The renormalisation group map. The renormalisation group approach of $[8,9]$ (and of several other authors as well) is based on a finite-range decomposition of the covariance $C=(\epsilon I-\Delta)^{-1}$, due to [4]. Fix a large integer $L$ and suppose that $|\Lambda|=L^{N d}$. Using the results of [4], it is possible to write

$$
C=\sum_{j=1}^{N} C_{j}
$$

where the $C_{j}$ 's are positive semi-definite operators with the important finite-range property

$$
C_{j}(x, y)=0 \quad \text { if } \quad|x-y| \geq L^{j} .
$$

The $C_{j}$ 's also have a certain self-similarity property, and obey the estimates

$$
\sup _{x, y} \sup _{|\alpha| \leq \alpha_{0}}\left|\nabla_{x}^{\alpha} \nabla_{y}^{\alpha} C_{j}(x, y)\right| \leq \operatorname{const} L^{-2 j} L^{-2(j-1)|\alpha|}
$$

with a $j$-independent constant. This decomposition induces a field decomposition

$$
\varphi=\sum_{j=1}^{N} \zeta_{j}, \quad d \varphi=\sum_{j=1}^{N} d \zeta_{j},
$$

and allows the expectation to be performed iteratively:

$$
\mathbb{E}_{C}=\mathbb{E}_{C_{N}} \circ \cdots \circ \mathbb{E}_{C_{2}} \circ \mathbb{E}_{C_{1}},
$$

where $\mathbb{E}_{C_{j}}$ integrates out the scale- $j$ fields $\zeta_{j}, \bar{\zeta}_{j}, d \zeta_{j}, d \bar{\zeta}_{j}$. Under $\mathbb{E}_{j}$, the scale- $j$ fields are uncorrelated when separated by distance greater than $L^{j}$, in contrast to the long-range correlations of the full expectation $\mathbb{E}_{C}$.

In what follows, we discuss the approach of [9] towards a direct evaluation of the integral $\int e^{-S} e^{-V(\Lambda)}$. In fact, as already pointed out above, it is a consequence of supersymmetry that this integral is equal to 1 , so direct evaluation is not necessary. However, the method described below extends also to evaluate the integral in (7.16), and it is easier to discuss the method now in the simpler setting without the factor $\bar{\varphi}_{x} \varphi_{y}$ in the integrand. 
We write $(\phi, d \phi)=(\varphi, \bar{\varphi}, d \varphi, d \bar{\varphi})$ and $(\xi, d \xi)=(\zeta, \bar{\zeta}, d \zeta, d \bar{\zeta})$. We set $\phi_{j}=$ $\sum_{i=j+1}^{N} \xi_{i}$, with $\phi_{0}=\phi, \phi_{N}=0$; this gives

$$
\phi_{j}=\phi_{j+1}+\xi_{j+1} .
$$

Let $Z_{0}=Z_{0}(\phi, d \phi)=e^{-V(\Lambda)}$, let

$$
\begin{aligned}
& Z_{1}\left(\phi_{1}, d \phi_{1}\right)=\mathbb{E}_{C_{1}} Z_{0}\left(\phi_{1}+\xi_{1}, d \phi_{1}+d \xi_{1}\right), \\
& Z_{2}\left(\phi_{2}, d \phi_{2}\right)=\mathbb{E}_{C_{2}} Z_{1}\left(\phi_{2}+\xi_{2}, d \phi_{2}+d \xi_{2}\right)=\mathbb{E}_{C_{2}} \mathbb{E}_{C_{1}} Z_{0},
\end{aligned}
$$

and, in general, let

$$
Z_{j}\left(\phi_{j}, d \phi_{j}\right)=\mathbb{E}_{C_{j}} \cdots \mathbb{E}_{C_{1}} Z_{0}(\phi, d \phi) .
$$

Our goal now is to compute directly

$$
Z_{N}=\mathbb{E}_{C} Z_{0}=\mathbb{E}_{C} e^{-V(\Lambda)}
$$

This leads us to study the renormalisation group map $Z_{j} \mapsto Z_{j+1}$ given by

$$
Z_{j+1}\left(\phi_{j+1}, d \phi_{j+1}\right)=\mathbb{E}_{C_{j+1}} Z_{j}\left(\phi_{j+1}+\xi_{j+1}, d \phi_{j+1}+d \xi_{j+1}\right) .
$$

The finite-range property of $C_{j}$, together with our choice of side length $L^{N}$ for $\Lambda$, leads naturally to the consideration of $\Lambda$ as being paved by blocks of side $L^{j}$. Let $\mathcal{P}_{j}$ denote the set of finite unions of such blocks. Given forms $F, G$ defined on $\mathcal{P}_{j}$, we define the product

$$
(F \circ G)(\Lambda)=\sum_{X \in \mathcal{P}_{j}} F(X) G(\Lambda \backslash X) .
$$

For $X \in \mathcal{P}_{0}$, let

$$
I_{0}(X)=e^{-V(X)}, \quad K_{0}(X)=\mathbb{1}_{X=\varnothing .}
$$

Then we can write

$$
Z_{0}=I_{0}(\Lambda)=\left(I_{0} \circ K_{0}\right)(\Lambda) .
$$

The method of [9] consists in the determination of an inductive parametrisation

$$
Z_{j}=\left(I_{j} \circ K_{j}\right)(\Lambda), \quad Z_{j+1}=\mathbb{E}_{C_{j+1}} Z_{j}=\left(I_{j+1} \circ K_{j+1}\right)(\Lambda),
$$

with each $I_{j}$ parametrised in turn by a polynomial $V_{j}$ evaluated at $\phi_{j}, d \phi_{j}$, given by

$$
V_{j, x}=g_{j} \tau_{x}^{2}+\lambda_{j} \tau_{x}+z_{j} \tau_{\Delta, x}
$$

with

$$
\tau_{\Delta, x}=\varphi_{x}(-\Delta \bar{\varphi})_{x}+(-\Delta \varphi)_{x} \bar{\varphi}_{x}+d \varphi_{x}(-\Delta d \bar{\varphi})_{x}+(-\Delta d \varphi)_{x} d \bar{\varphi}_{x} .
$$

The term $K_{j}$ accumulates error terms. The map $I_{j} \mapsto I_{j+1}$ is thus given by the flow of the coupling constants $\left(g_{j}, \lambda_{j}, z_{j}\right) \mapsto\left(g_{j+1}, \lambda_{j+1}, z_{j+1}\right)$, and hence the renormalisation group map becomes the dynamical system

$$
\left(g_{j}, \lambda_{j}, z_{j}, K_{j}\right) \mapsto\left(g_{j+1}, \lambda_{j+1}, z_{j+1}, K_{j+1}\right) .
$$

At the critical point, this dynamical system is driven to zero, and this permits the asymptotic computation of the two-point function. 


\section{Dimensions $d \geq 5$}

8.1. Results. The following theorem shows that above the upper critical dimension the self-avoiding walk behaves like simple random walk, in the sense that $\gamma=1, \nu=\frac{1}{2}, \eta=0$, and the scaling limit is Brownian motion.

Theorem 8.1. [22, 23] For $d \geq 5$, there are positive constants $A, D, c, \epsilon$ such that

$$
\begin{aligned}
c_{n} & =A \mu^{n}\left[1+O\left(n^{-\epsilon}\right)\right], \\
\mathbb{E}_{n}|\omega(n)|^{2} & =\operatorname{Dn}\left[1+O\left(n^{-\epsilon}\right)\right],
\end{aligned}
$$

and the rescaled self-avoiding walk converges weakly to Brownian motion:

$$
\frac{\omega(\lfloor n t\rfloor)}{\sqrt{D n}} \Rightarrow B_{t}
$$

Also [20], as $|x| \rightarrow \infty$,

$$
G_{z_{c}}(x)=c|x|^{-(d-2)}\left[1+O\left(|x|^{-\epsilon}\right)\right] .
$$

The proofs of these results are based on the lace expansion, a technique that was introduced by Brydges and Spencer [10] to study the weakly self-avoiding walk in dimensions $d>4$. Since 1985, the method has been highly developed and extended to several other models: percolation $(d>6)$, oriented percolation $(d+1>4+1)$, contact process $(d>4)$, lattice trees and lattice animals $(d>8)$, and the Ising model $(d>4)$. For a review and references, see [49].

The lace expansion requires a small parameter for its convergence. For the nearest-neighbour model in dimensions $d \geq 5$, the small parameter is proportional to $(d-4)^{-1}$, which is not very small when $d=5$. Because of this, the proof of Theorem 8.1 is computer assisted. The weakly self-avoiding walk has an intrinsic small parameter $g$, and it is therefore easier to analyse than the strictly self-avoiding walk. Another option for the introduction of a small parameter is to consider the spread-out strictly self-avoiding walk, which takes steps within a box of side length $L$ centred at its current position; this model also can be more easily analysed by taking $L$ large to provide a small parameter $L^{-1}$.

The spread-out model can be generalised to have non-uniform step weights. For example, given $\alpha>0$, and an $n$-step self-avoiding walk $\omega$ on $\mathbb{Z}^{d}$ taking arbitrary steps, we define the weight

$$
W(\omega)=\prod_{i=1}^{n} \frac{1}{\left(L^{-1}|\omega(i-1)-\omega(i)| \wedge 1\right)^{d+\alpha}},
$$

and consider the probability distribution on self-avoiding walks that corresponds to this weight. The following theorem, which is proved using the lace expansion, shows how the upper critical dimension changes once $\alpha \leq 2$ and the step weights have infinite variance. 
Theorem 8.2. [24] Let $\alpha>0$ and

$$
f_{\alpha}(n)=a_{\alpha} \begin{cases}n^{-1 /(\alpha \wedge 2)} & \alpha \neq 2 \\ (n \log n)^{-1 / 2} & \alpha=2,\end{cases}
$$

for a suitably chosen (explicit) constant $a_{\alpha}$. For $d>2(\alpha \wedge 2)$ and for $L$ sufficiently large, the process $X_{n}(t)=f_{\alpha}(n) \omega(\lfloor n t\rfloor)$ converges in distribution to an $\alpha$-stable Lévy process if $\alpha<2$ and to Brownian motion if $\alpha \geq 2$.

In [43], the weakly self-avoiding walk with long-range steps characterised by $\alpha=\frac{3+\epsilon}{2}$, with small $\epsilon>0$, is studied in dimension 3, which is below the upper critical dimension $3+\epsilon$. The main result is the control of the renormalisation group trajectory, a first step towards the computation of the asymptotics for the critical two-point function below the upper critical dimension. This is a rigorous version, for the weakly self-avoiding walk, of the expansion in $\epsilon=4-d$ discussed in [51]. The work of [43] is based on the functional integral representation outlined in Section 7.4.

8.2. The lace expansion. The original formulation of the lace expansion in [10] made use of a particular class of ordered graphs which Brydges and Spencer called "laces." Later it was realised that the same expansion can be obtained by repeated use of inclusion-exclusion [48]. We now sketch the inclusion-exclusion approach very briefly; further details can be found in [40] or [49].

The lace expansion identifies a function $\pi_{m}(x)$ such that for $n \geq 1$,

$$
c_{n}(x)=\sum_{y \in \mathbb{Z}^{d}} c_{1}(y) c_{n-1}(x-y)+\sum_{m=2}^{n} \sum_{y \in \mathbb{Z}^{d}} \pi_{m}(y) c_{n-m}(x-y) .
$$

In fact, it is possible to see that (8.7) defines $\pi_{m}(x)$, but the expansion will produce a useful expression for $\pi_{m}(x)$. We begin with the identity

$$
c_{n}(x)=\sum_{y \in \mathbb{Z}^{d}} c_{1}(y) c_{n-1}(x-y)-R_{n}^{(1)}(x),
$$

where $R_{n}^{(1)}(x)$ counts the number of terms which are included on the first term of the right-hand side but excluded on the left, namely the number of $n$-step walks which start at 0 , end at $x$, and are self-avoiding except for an obligatory single return to 0 . This is denoted schematically by

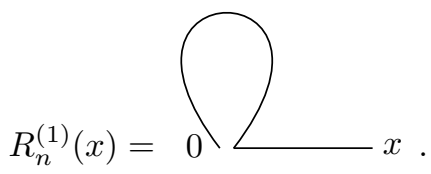

If we relax the constraint that the loop in the above diagram avoid the vertices in the "tail," then we are led to

$$
R_{n}^{(1)}(x)=\sum_{m=2}^{n} u_{m} c_{n-m}(x)-R_{n}^{(2)}(x)
$$


where $u_{m}$ is the number of $m$-step self-avoiding returns, and

$$
R_{n}^{(2)}(x)=0\{\text { simm } x \text {. }
$$

In the above diagram, the proper line represents a self-avoiding return, while the wavy line represents a self-avoiding walk from 0 to $x$ constrained to intersect the proper line. Repetition of the inclusion-exclusion process leads to

$$
c_{n}(x)=\sum_{y \in \mathbb{Z}^{d}} c_{1}(y) c_{n-1}(x-y)+\sum_{m=2}^{n} \sum_{y \in \mathbb{Z}^{d}} \pi_{m}(y) c_{n-m}(x-y)
$$

with

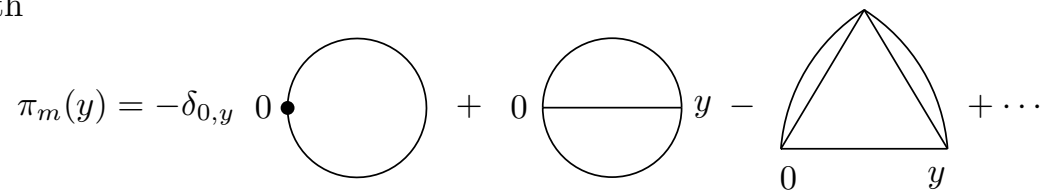

and where there are specific rules for which lines may intersect which in the diagrams on the right-hand side. These rules can be conveniently accounted for using the concept of lace.

We put (8.7) into a generating function to obtain

$$
G_{z}(x)=\sum_{n=0}^{\infty} c_{n}(x) z^{n}=\delta_{0, x}+z \sum_{y \in \mathbb{Z}^{d}} c_{1}(y) G_{z}(x-y)+\sum_{y \in \mathbb{Z}^{d}} \Pi_{z}(y) G_{z}(x-y)
$$

with

$$
\Pi_{z}(y)=\sum_{m=2}^{\infty} \pi_{m}(y) z^{m}
$$

For $k \in[-\pi, \pi]^{d}$, let $\hat{f}(k)=\sum_{x} f(x) e^{i k \cdot x}$ denote the Fourier transform of an absolutely summable function $f$ on $\mathbb{Z}^{d}$. From (8.14), we obtain

$$
\hat{G}_{z}(k)=\frac{1}{1-z \hat{c}_{1}(k)-\hat{\Pi}_{z}(k)} .
$$

Note that setting $\hat{\Pi}_{z}(k)$ equal to zero yields the Fourier transform of the two-point function of simple random walk, and hence $\hat{\Pi}_{z}(k)$ encapsulates the self-avoidance.

8.3. One idea from the proof of Theorem 8.1. Let $\hat{F}_{z}(k)=1 / \hat{G}_{z}(k)$. By definition, $\hat{G}_{z}(0)=\sum_{n=0}^{\infty} c_{n} z^{n}$. Since $\lim _{n \rightarrow \infty} c_{n}^{1 / n}=\mu=z_{c}^{-1}, \hat{G}_{z}(0)$ has radius of convergence $z_{c}$, and since $c_{n} \geq \mu^{n}, \hat{F}_{z_{c}}(0)=0$. Suppose that it is possible to 
perform a joint Taylor expansion of $\hat{F}$ in $k$ and $z$ about the points $k=0$ and $z=z_{c}$. The linear term in $k$ vanishes by symmetry, so that

$$
\hat{F}_{z}(k)=\hat{F}_{z}(k)-\hat{F}_{z_{c}}(0) \approx a|k|^{2}+b\left(1-\frac{z}{z_{c}}\right), \quad \text { for } k \approx 0, z \approx z_{c}^{-},
$$

with $a=\frac{1}{2 d} \nabla_{k}^{2} \hat{F}_{z_{c}}(0)$ and $b=-z_{c} \partial_{z} \hat{F}_{z_{c}}(0)$. We assume now that $a$ and $b$ are finite, although it is an important part of the proof to establish this, and it is not expected to be true when $d \leq 4$. Then

$$
\hat{G}_{z}(k) \approx \frac{1}{a|k|^{2}+b\left(1-\frac{z}{z_{c}}\right)}, \quad \text { for } k \approx 0, z \approx z_{c}^{-},
$$

which is essentially the corresponding generating function for simple random walk.

For this to work, it is necessary in particular that $z_{c} \partial_{z} \hat{\Pi}_{z_{c}}(k)$ be finite. The leading term in this derivative, due to the first term $\sum_{m=2}^{\infty} u_{m} z^{m}$ in the diagrammatic expansion for $\hat{\Pi}_{z}(k)$, is $\sum_{m=2}^{\infty} m u_{m} z_{c}^{m}$. By considering the factor $m$ to be the number of ways to choose a nonzero vertex on a self-avoiding return, and by relaxing the constraint that the two parts of this return (separated by the chosen vertex) avoid each other, we find that this contribution is bounded above by

$$
\sum_{m=2}^{\infty} u_{m} m z_{c}^{m} \leq \sum_{x \in \mathbb{Z}^{d}} G_{z_{c}}(x)^{2}=\int_{[-\pi, \pi]^{d}} \hat{G}_{z_{c}}(k)^{2} \frac{d^{d} k}{(2 \pi)^{d}},
$$

where the equality follows from Parseval's relation. A reason to be hopeful that this might lead to a finite upper bound is that if we insert the simple random walk behaviour on the right-hand side of (8.18) into (8.19) then we obtain

$$
\int_{[-\pi, \pi]^{d}} \hat{G}_{z_{c}}(k)^{2} \frac{d^{d} k}{(2 \pi)^{d}} \approx \int_{[-\pi, \pi]^{d}} \frac{1}{|k|^{4}} \frac{d^{d} k}{(2 \pi)^{d}}<\infty \quad \text { for } d>4 .
$$

Here we have assumed what it is that we are trying to prove, but the proof finds a way to exploit this kind of self-consistent argument. For the details, we refer to $[22,23]$, or, in the much simpler setting of the spread-out model, to [49].

\section{Conclusions}

Our current understanding of the critical behaviour of the self-avoiding walk can be summarised as follows:

- $d=1$ : ballistic behaviour is trivial for the nearest-neighbour strictly selfavoiding walk, but is interesting for the weakly self-avoiding walk.

- $d=2$ : if the scaling limit can be proven to exist and to be conformally invariant then the scaling limit is $\mathrm{SLE}_{8 / 3}, \mathrm{SLE}_{8 / 3}$ explains the values $\gamma=\frac{43}{32}$ and $\nu=\frac{3}{4}$, currently there is no proof that the scaling limit exists. 
- $d=3:$ numerically $\gamma \approx 1.16$ and $\nu \approx 0.588$, there are no rigorous results, and there is no idea how to describe the scaling limit as a stochastic process.

- $d=4$ : renormalisation group methods have proved that $\eta=0$ for continuoustime weakly self-avoiding walk; on a 4-dimensional hierarchical lattice $\gamma=1$ and $\nu=\frac{1}{2}$, both with log corrections, and $\eta=0$.

- $d \geq 5$ : the problem is solved using the lace expansion, $\gamma=1, \nu=\frac{1}{2}, \eta=0$, and the scaling limit is Brownian motion.

\section{Acknowledgements}

The discussion in Section 7.5 is based on work in progress with David Brydges, to whom I am grateful for helpful comments on a preliminary version of this article. I thank Roland Bauerschmidt and Takashi Hara for their help with the figures. The hospitality of the Institut Henri Poincaré, where this article was written, is gratefully acknowledged.

\section{References}

[1] E. Brézin, J.C. Le Guillou, and J. Zinn-Justin. Approach to scaling in renormalized perturbation theory. Phys. Rev. D, 8:2418-2430, (1973).

[2] D.C. Brydges. Lectures on the renormalisation group. In S. Sheffield and T. Spencer, editors, Statistical Mechanics, pages 7-93. American Mathematical Society, Providence, (2009). IAS/Park City Mathematics Series, Volume 16.

[3] D. Brydges, S.N. Evans, and J.Z. Imbrie. Self-avoiding walk on a hierarchical lattice in four dimensions. Ann. Probab., 20:82-124, (1992).

[4] D.C. Brydges, G. Guadagni, and P.K. Mitter. Finite range decomposition of Gaussian processes. J. Stat. Phys., 115:415-449, (2004).

[5] D.C. Brydges and J.Z. Imbrie. End-to-end distance from the Green's function for a hierarchical self-avoiding walk in four dimensions. Commun. Math. Phys., 239:523547, (2003).

[6] D.C. Brydges and J.Z. Imbrie. Green's function for a hierarchical self-avoiding walk in four dimensions. Commun. Math. Phys., 239:549-584, (2003).

[7] D.C. Brydges, J.Z. Imbrie, and G. Slade. Functional integral representations for self-avoiding walk. Probab. Surveys, 6:34-61, (2009).

[8] D. Brydges and G. Slade. Renormalisation group analysis of weakly self-avoiding walk in dimensions four and higher. To appear in Proceedings of the International Congress of Mathematicians, Hyderabad, 2010, ed. R. Bhatia, Hindustan Book Agency, Delhi.

[9] D.C. Brydges and G. Slade. Papers in preparation.

[10] D.C. Brydges and T. Spencer. Self-avoiding walk in 5 or more dimensions. Commun. Math. Phys., 97:125-148, (1985). 
[11] S. Caracciolo, M.S. Causo, and A. Pelissetto. High-precision determination of the critical exponent $\gamma$ for self-avoiding walks. Phys. Rev. E, 57:1215-1218, (1998).

[12] N. Clisby. Accurate estimate of the critical exponent $\nu$ for self-avoiding walks via a fast implementation of the pivot algorithm. Phys. Rev. Lett., 104:055702, (2010).

[13] N. Clisby, R. Liang, and G. Slade. Self-avoiding walk enumeration via the lace expansion. J. Phys. A: Math. Theor., 40:10973-11017, (2007).

[14] P.J. Flory. The configuration of a real polymer chain. J. Chem. Phys., 17:303-310, (1949).

[15] P.G. de Gennes. Exponents for the excluded volume problem as derived by the Wilson method. Phys. Lett., A38:339-340, (1972).

[16] S.E. Golowich and J.Z. Imbrie. The broken supersymmetry phase of a self-avoiding random walk. Commun. Math. Phys., 168:265-319, (1995).

[17] A. Greven and F. den Hollander. A variational characterization of the speed of a one-dimensional self-repellent random walk. Ann. Appl. Probab., 3:1067-1099, (1993).

[18] R. Guida and J. Zinn-Justin. Critical exponents of the N-vector model. J. Phys. A: Math. Gen., 31:8103-8121, (1998).

[19] J.M. Hammersley and D.J.A. Welsh. Further results on the rate of convergence to the connective constant of the hypercubical lattice. Quart. J. Math. Oxford, (2), 13:108-110, (1962).

[20] T. Hara. Decay of correlations in nearest-neighbor self-avoiding walk, percolation, lattice trees and animals. Ann. Probab., 36:530-593, (2008).

[21] T. Hara and M. Ohno. Renormalization group analysis of hierarchical weakly selfavoiding walk in four dimensions. In preparation.

[22] T. Hara and G. Slade. Self-avoiding walk in five or more dimensions. I. The critical behaviour. Commun. Math. Phys., 147:101-136, (1992).

[23] T. Hara and G. Slade. The lace expansion for self-avoiding walk in five or more dimensions. Reviews Math. Phys., 4:235-327, (1992).

[24] M. Heydenreich. Long-range self-avoiding walk converges to alpha-stable processes. To appear in Ann. I. Henri Poincaré Probab. Statist. Preprint, (2008).

[25] R. van der Hofstad. The lace expansion approach to ballistic behaviour for onedimensional weakly self-avoiding walk. Probab. Theory Related Fields, 119:311-349, (2001).

[26] R. van der Hofstad and W. König. A survey of one-dimensional random polymers. J. Stat. Phys., 103:915-944, (2001).

[27] F. den Hollander. Random Polymers. Springer, Berlin, (2009). Lecture Notes in Mathematics Vol. 1974. Ecole d'Eté de Probabilités de Saint-Flour XXXVII-2007.

[28] I. Horváth, B. Tóth, and B. Vető. Diffusive limit for the myopic (or "true") selfavoiding random walk in $d \geq 3$. Preprint, (2009).

[29] B.D. Hughes. Random Walks and Random Environments, volume 1: Random Walks. Oxford University Press, Oxford, (1995).

[30] E. J. Janse van Rensburg. Monte Carlo methods for the self-avoiding walk. J. Phys. A: Math. Theor., 42:323001, (2009). 
[31] I. Jensen. A parallel algorithm for the enumeration of self-avoiding polygons on the square lattice. J. Phys. A: Math. Gen., 36:5731-5745, (2003).

[32] I. Jensen. Enumeration of self-avoiding walks on the square lattice. J. Phys. A: Math. Gen., 37:5503-5524, (2004).

[33] I. Jensen. Improved lower bounds on the connective constants for two-dimensional self-avoiding walks. J. Phys. A: Math. Gen., 11521-11529, (2004).

[34] T. Kennedy. Conformal invariance and stochastic Loewner evolution predictions for the 2D self-avoiding walk-Monte Carlo tests. J. Stat. Phys., 114:51-78, (2004).

[35] H. Kesten. On the number of self-avoiding walks. II. J. Math. Phys., 5:1128-1137, (1964).

[36] W. König. A central limit theorem for a one-dimensional polymer measure. Ann. Probab, 24:1012-1035, (1996).

[37] G.F. Lawler, O. Schramm, and W. Werner. On the scaling limit of planar selfavoiding walk. Proc. Symposia Pure Math., 72:339-364, (2004).

[38] Y. Le Jan. Temps local et superchamp. In Séminaire de Probabilités XXI. Lecture Notes in Mathematics \#1247, pages 176-190, Berlin, (1987). Springer.

[39] B. Li, N. Madras, and A.D. Sokal. Critical exponents, hyperscaling, and universal amplitude ratios for two- and three-dimensional self-avoiding walks. J. Stat. Phys., 80:661-754, (1995).

[40] N. Madras and G. Slade. The Self-Avoiding Walk. Birkhäuser, Boston, (1993).

[41] N. Madras and A.D. Sokal. The pivot algorithm: A highly efficient Monte Carlo method for the self-avoiding walk. J. Stat. Phys., 50:109-186, (1988).

[42] A.J. McKane. Reformulation of $n \rightarrow 0$ models using anticommuting scalar fields. Phys. Lett. A, 76:22-24, (1980).

[43] P.K. Mitter and B. Scoppola. The global renormalization group trajectory in a critical supersymmetric field theory on the lattice $\mathbf{Z}^{3}$. J. Stat. Phys., 133:921-1011, (2008).

[44] B. Nienhuis. Exact critical exponents of the $O(n)$ models in two dimensions. Phys. Rev. Lett., 49:1062-1065, (1982).

[45] G. Parisi and N. Sourlas. Self-avoiding walk and supersymmetry. J. Phys. Lett., 41:L403-L406, (1980).

[46] A. Pönitz and P. Tittmann. Improved upper bounds for self-avoiding walks in $\mathbb{Z}^{d}$. Electron. J. Combin., 7:Paper R21, (2000).

[47] Y. Roiter and S. Minko. AFM single molecule experiments at the solid-liquid interface: In situ conformation of adsorbed flexible polyelectrolyte chains. J. Am. Chem. Soc., 45:15688-15689, (2005).

[48] G. Slade. The lace expansion and the upper critical dimension for percolation. Lectures in Applied Mathematics, 27:53-63, (1991). (Mathematics of Random Media, eds. W.E. Kohler and B.S. White, A.M.S., Providence).

[49] G. Slade. The Lace Expansion and its Applications. Springer, Berlin, (2006). Lecture Notes in Mathematics Vol. 1879. Ecole d'Eté de Probabilités de Saint-Flour XXXIV2004.

[50] B. Tóth and B. Vető. Continuous time 'true' self-avoiding walk on $Z$. Preprint, (2009). 
[51] K. Wilson and J. Kogut. The renormalization group and the $\epsilon$ expansion. Phys. Rep., 12:75-200, (1974).

Gordon Slade, Department of Mathematics, University of British Columbia, Vancouver, BC, Canada V6T 1 Z2

E-mail: slade@math.ubc.ca 